\title{
Evaluation of the maintenance of stemness, viability, and differentiation potential of gingiva-derived stem-cell spheroids
}

\author{
SUNG-IL LEE, YOUNGKYUNG KO and JUN-BEOM PARK \\ Department of Periodontics, College of Medicine, The Catholic University of Korea, Seoul 06591, Republic of Korea
}

Received December 11, 2015; Accepted December 23, 2016

DOI: 10.3892/etm.2017.4194

\begin{abstract}
Gingiva-derived stem cells have been applied for tissue-engineering purposes and may be considered a favorable source of mesenchymal stem cells as harvesting stem cells from the mandible or maxilla may be performed with ease under local anesthesia. The present study was performed to fabricate stem-cell spheroids using concave microwells and to evaluate the maintenance of stemness, viability, and differentiation potential. Gingiva-derived stem cells were isolated, and the stem cells of $4 \times 10^{5}$ (group A) or $8 \times 10^{5}$ (group B) cells were seeded into polydimethylsiloxane-based, concave micromolds with $600 \mu \mathrm{m}$ diameters. The morphology of the microspheres and the change of the diameters of the spheroids were evaluated. The viability of spheroids was qualitatively analyzed via Live/Dead kit assay. A cell viability analysis was performed on days 1, 3, 6, and 12 with Cell Counting Kit-8. The maintenance of stemness was evaluated with immunocytochemical staining using SSEA-4, TRA-1-60(R) (positive markers), and SSEA-1 (negative marker). Osteogenic, adipogenic, and chondrogenic differentiation potential was evaluated by incubating spheroids in osteogenic, adipogenic and chondrogenic induction medium, respectively. The gingiva-derived stem cells formed spheroids in the concave microwells. The diameters of the spheroids were larger in group A than in group B. The majority of cells in the spheroids emitted green fluorescence, indicating the presence of live cells at day 6 . At day 12, the majority of cells in the spheroids emitted green fluorescence, and a small portion of red fluorescence was also noted, which indicated the presence of dead cells. The spheroids were positive for the stem-cell markers SSEA-4 and TRA-1-60(R) and were negative for SSEA-1, suggesting that these spheroids primarily contained undifferentiated human stem cells. Osteogenic, adipogenic, and chondrogenic differentiation was more evident with an increase of incubation time: Mineralized
\end{abstract}

Correspondence to: Dr Jun-Beom Park, Department of Periodontics, College of Medicine, The Catholic University of Korea, 222 Banpo-daero, Seocho-gu, Seoul 06591, Republic of Korea E-mail: jbassoonis@yahoo.co.kr

Key words: cell culture techniques, cellular spheroids, cell survival, stem cell research, stem cells extracellular deposits were observed following Alizarin Red S staining at days 14 and 21; oil globules were increased at day 18 when compared with day 6; and Alcian blue staining was more evident at day 18 when compared with day 6 . Within the limits of this study, stem-cell spheroids from gingival cells maintained the stemness, viability, and differentiation potential during the experimental periods. This method may be applied for a promising strategy for stem-cell therapy.

\section{Introduction}

Mesenchymal stem cells are clonogenic, self-renewing progenitor cells that are able to generate one or more specialized cell types (1). Stem cells may be postnatally isolated from different tissues including adipose tissue, muscle, bone marrow, and periosteum (2). Mesenchymal stem cells are a valuable source of stem cells for regenerative medicine, and the transplantation of mesenchymal stem cells is a promising treatment for many diseases $(3,4)$. However, conventional techniques, which consist of cells being cultured as a monolayer, typically result in slow cell proliferation and insufficient yield to fulfill clinical demands (3). Furthermore, the loss of stemness, which is the ability to self-renew and differentiate, of mesenchymal stem cells during in vitro expansion reduces the therapeutic efficacy (4).

Three-dimensional culture systems have been used to demonstrate the importance of intercellular interactions in regulating stem cell self-renewal and differentiation (5). Due to their rich biological content and superior ability to mimic the in vivo environment compared with two-dimensional cell cultures, such multi-cellular spheroids are currently receiving increased attention with regard to their applications and production (6). Typically, cells in a spheroid culture exhibit various properties that are distinct from monolayer cells (7). Cells grow with similar characteristics to in vivo tissue and these cultures are able to simulate native tissue behaviors much more accurately than two-dimensional cultures (7). A recent study has demonstrated that the stemness properties of mesenchymal stem cells are retained in the in vivo microenvironment, which comprises cell-cell interactions, soluble growth factors, and cell-matrix interactions (4).

Human mesenchymal stem cells have been isolated and characterized from the periodontium including the gingiva (8), and gingiva-derived stem cells have been utilized for tissue-engineering purposes (9). Gingiva-derived stem cells 
from the maxillofacial region may be considered a favorable source of mesenchymal stem cells as harvesting stem cells from the mandible or maxilla may be easily performed under local anesthesia $(8,10)$. The present study was performed to produce stem-cell spheroids using concave microwells and to evaluate the maintenance of stemness, viability, and differentiation potential. To the best of our knowledge, this study is the first to evaluate the maintenance of stemness, viability, and differentiation potential of gingiva-derived stem-cell spheroids.

\section{Materials and methods}

Isolation and culturing of gingiva-derived stem cells. Gingiva-derived stem cells and cultures were obtained using a previously reported method (8). Gingival tissues were harvested from healthy patients during crown lengthening procedures from July 2013 to August 2015 visiting the Department of Periodontics, Seoul St. Mary's Hospital. Exclusion criteria were as follows: i) Severe medical or psychological disease or ii) hemorrhagic disease. The design of the present study was reviewed and approved by the Institutional Review Board of Seoul St. Mary's Hospital, College of Medicine, Catholic University of Korea (Seoul, Korea; KC11SISI0348) and informed consent was obtained from the patients.

Attached keratinized gingival tissues were immediately placed in sterile phosphate-buffered saline (PBS; Welgene, Daegu, South Korea) with $100 \mathrm{U} / \mathrm{ml}$ penicillin and $100 \mu \mathrm{g} / \mathrm{ml}$ streptomycin (Sigma-Aldrich; Merck Millipore, Darmstadt, Germany) at $4^{\circ} \mathrm{C}$ until use. The tissues were de-epithelialized with a surgical blade, processed into $1-2 \mathrm{~mm}^{2}$ fragments, and digested in an $\alpha$-modified, minimal essential medium ( $\alpha$-MEM; Gibco; Thermo Fisher Scientific, Inc., Waltham, MA, USA) containing dispase $(1 \mathrm{mg} / \mathrm{ml})$ and collagenase IV $(2 \mathrm{mg} / \mathrm{ml}$; both from Sigma-Aldrich; Merck Millipore). The cells were incubated at $37^{\circ} \mathrm{C}$ in a humidified incubator with $5 \% \mathrm{CO}_{2}$ and $95 \% \mathrm{O}_{2}$ for $24 \mathrm{~h}$. Non-adherent cells were subsequently washed with PBS (Welgene), replaced with a fresh medium ( $\alpha$-MEM) containing fetal bovine serum (both from Gibco), and penicillin, streptomycin and ascorbic acid 2-phosphate (all from Sigma-Aldrich; Merck Millipore) every 2 to 3 days.

Formation of spheres. Stem-cell spheroids were formed in silicon elastomer-based concave microwells (StemFIT 3D; MicroFIT, Seonnam, Republic of Korea) with $600 \mu \mathrm{m}$ diameters. Subsequently, $4 \times 10^{5}$ (group A) or $8 \times 10^{5}$ (group B) stem cells were seeded in each concave micromold and cultured to investigate cellular behavior (Fig. 1). The difference between group A and group B was only the number of cells per spheroid. Cell aggregation and spheroid formation were observed and images were captured using an inverted microscope (Leica DM IRM; Leica Microsystems GmbH, Wetzlar, Germany). The diameters of spheroids were measured from the captured images.

Determination of cell viability. Viability of spheroids was qualitatively analyzed using a Live/Dead assay kit (Molecular Probes; Thermo Fisher Scientific, Inc.). The assay is based on the principle that the activity of intracellular esterase induces non-fluorescent, cell-permeant calcein acetoxymethyl to become intensely fluorescent, giving the viable spheroids a green fluorescence. Ethidium homodimer enters into damaged cell membrane and binds to nucleic acids, thereby producing a red fluorescence in dead cells.

Stem-cell spheroids were cultured at $37^{\circ} \mathrm{C}$ in $\alpha$-MEM containing $15 \%$ fetal bovine serum (Gibco; Thermo Fisher Scientific, Inc.), $100 \mathrm{U} / \mathrm{ml}$ penicillin, $100 \mu \mathrm{g} / \mathrm{ml}$ streptomycin, $200 \mathrm{mM}$ L-glutamine and $10 \mathrm{mM}$ ascorbic acid 2-phosphate (all from Sigma-Aldrich; Merck Millipore). These spheroids were washed twice with PBS, followed by suspension in $1 \mathrm{ml}$ $\alpha$-MEM containing $2 \mu \mathrm{l} 50 \mathrm{mM}$ calcein acetoxymethyl ester working solution and $4 \mu 12 \mathrm{mM}$ ethidium homodimer-1 for $15 \mathrm{~min}$ at room temperature. The spheroids stained with calcein acetoxymethyl ester and ethidium homodimer-1 were observed under a fluorescence microscope (Axiovert 200; Zeiss AG, Oberkochen, Germany) at days 6 and 12 .

A cell-viability analysis was performed on days 1, 3, 6, and 12 , by adding 2-(2-methoxy-4-nitrophenyl)-3-(4-nitrop henyl)-5-(2,4-disulfophenyl)-2H tetrazolium monosodium salt (WST-8; Cell Counting Kit-8; Dojindo Molecular Technologies, Inc., Kumamoto, Japan) to cultures, and spheres were incubated for $1 \mathrm{~h}$ at $37^{\circ} \mathrm{C}$. Viable cells were identified by the assay, which relies on the ability of mitochondrial dehydrogenases to oxidize WST-8 into a formazan product. The spectrophotometric absorbance of the samples was measured using a microplate reader (BioTek Instruments, Inc., Winooski, VT, USA) at $450 \mathrm{~nm}$.

Evaluation of maintenance of stemness. Following cultivation for six days, spheroids were retrieved. Antibodies were purchased from R\&D Systems, Inc., (Minneapolis, MN, USA), and diluted to 50X. Human SSEA-4 conjugated to NHL493 (green) and human TRA-1-60(R) conjugated to NL557 (red) antibodies (cat. no. SC023; Live Cell Imaging kit) were used as positive markers of human stem cells. Human SSEA-1 conjugated to NL557 (red) antibody (cat. no. SC023; Live Cell Imaging kit) was used as a negative marker. The cells were incubated for $30 \mathrm{~min}$. The antibody-containing media were removed, and cells were washed with fresh media and re-fed with fresh media. The spheroids were visualized via fluorescence microscopy.

Osteogenic differentiation. Spheroids were grown at $37^{\circ} \mathrm{C}$ in osteogenic induction medium (STEMPRO Osteogenesis Differentiation kit; Gibco; Thermo Fisher Scientific, Inc.). The medium was replaced with a fresh induction medium every 3 to 4 days. At days 14 and 21, Alizarin Red S staining (Sigma-Aldrich; Merck Millipore) was performed to detect calcium formation. Bound dye was solubilized in $10 \mathrm{mM}$ sodium phosphate containing $10 \%$ cetylpyridinium chloride and quantified spectrophotometrically at $562 \mathrm{~nm}$. The morphological evaluation was performed using an inverted microscope (Leica DM IRM) and experiments were performed in triplicate.

Adipogenic differentiation. Spheroids were grown at $37^{\circ} \mathrm{C}$ with adipogenic induction medium (STEMPRO Adipogenesis Differentiation kit; Gibco; Thermo Fisher Scientific, Inc.). The medium was replaced with a fresh induction medium every 3 to 4 days. Oil Red O staining (Sigma-Aldrich; Merck Millipore) was performed at days 6 and 18 to detect the oil globules. 


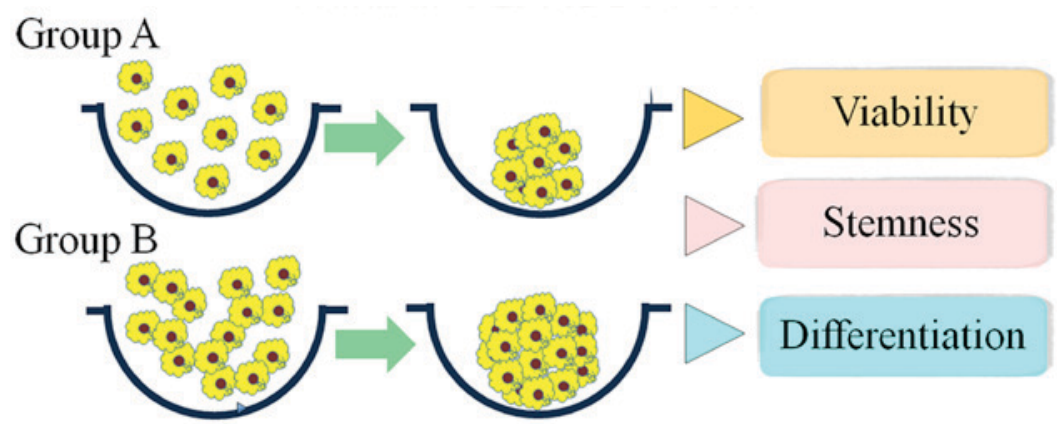

Figure 1. Schematic illustration of generation of the spheroids with gingival-derived stem cells.
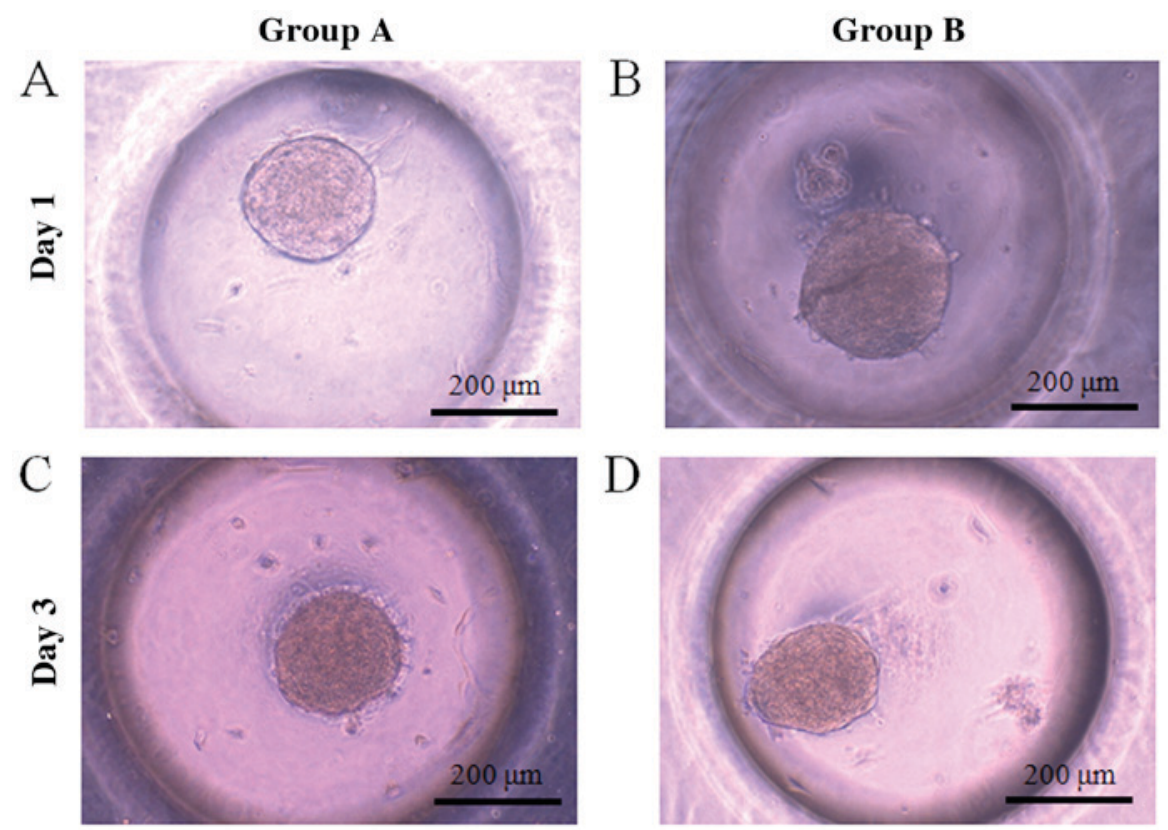

Figure 2. The morphology of the stem-cell spheroids at days 1 and 3. The scale bar indicates $200 \mu \mathrm{m}$ (original magnification, x200). (A) Group A at day 1; (B) group B at day 1; (C) group A at day 3; (D) group B at day 3.

Chondrogenic differentiation. Spheroids were grown at $37^{\circ} \mathrm{C}$ in a chondrogenic induction medium (STEMPRO Chondrogenesis Differentiation kit; Gibco). The medium was replaced with a fresh induction medium every 3 to 4 days. Alcian blue staining (Sigma-Aldrich; Merck Millipore) was performed following 14 days to detect the presence of cartilage-specific proteoglycan core protein.

Statistical analysis. Data are presented as means \pm standard deviations of the experiments. A test of normality using a Shapiro-Wilk test was performed and a Student's $t$-test or a two-way analysis of variance with post hoc Tukey testing was performed to determine the differences between the groups using SPSS 12 (SPSS, Inc., Chicago, IL, USA). P<0.05 was considered to indicate a statistically significant difference.

\section{Results}

Evaluation of cell morphology. Gingiva-derived stem cells were able to form spheroids in concave microwells. The morphology of the spheroids at day 1 is shown in Fig. 2A and B. The morphology of the spheroids at day 3 was similar to that

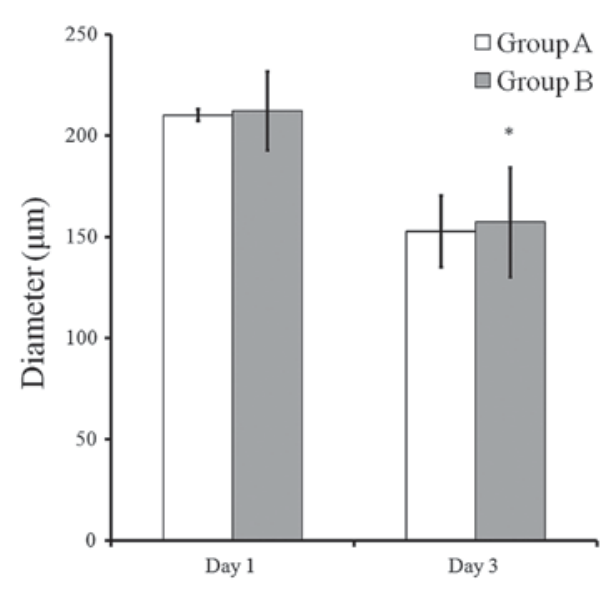

Figure 3. The mean diameter ( \pm standard deviation) of spheroids at days 1 and $3 .{ }^{*} \mathrm{P}<0.05$ vs. group $\mathrm{B}$ at day 1 .

of day 1 (Fig. 2C-D). The diameters of spheroids in group B were larger compared to those of group A. The mean spheroid diameters in group A were 210.0 \pm 3.0 and $152.5 \pm 17.8 \mu \mathrm{m}$ at days 1 and 3 , respectively (Fig. 3). The mean spheroid 


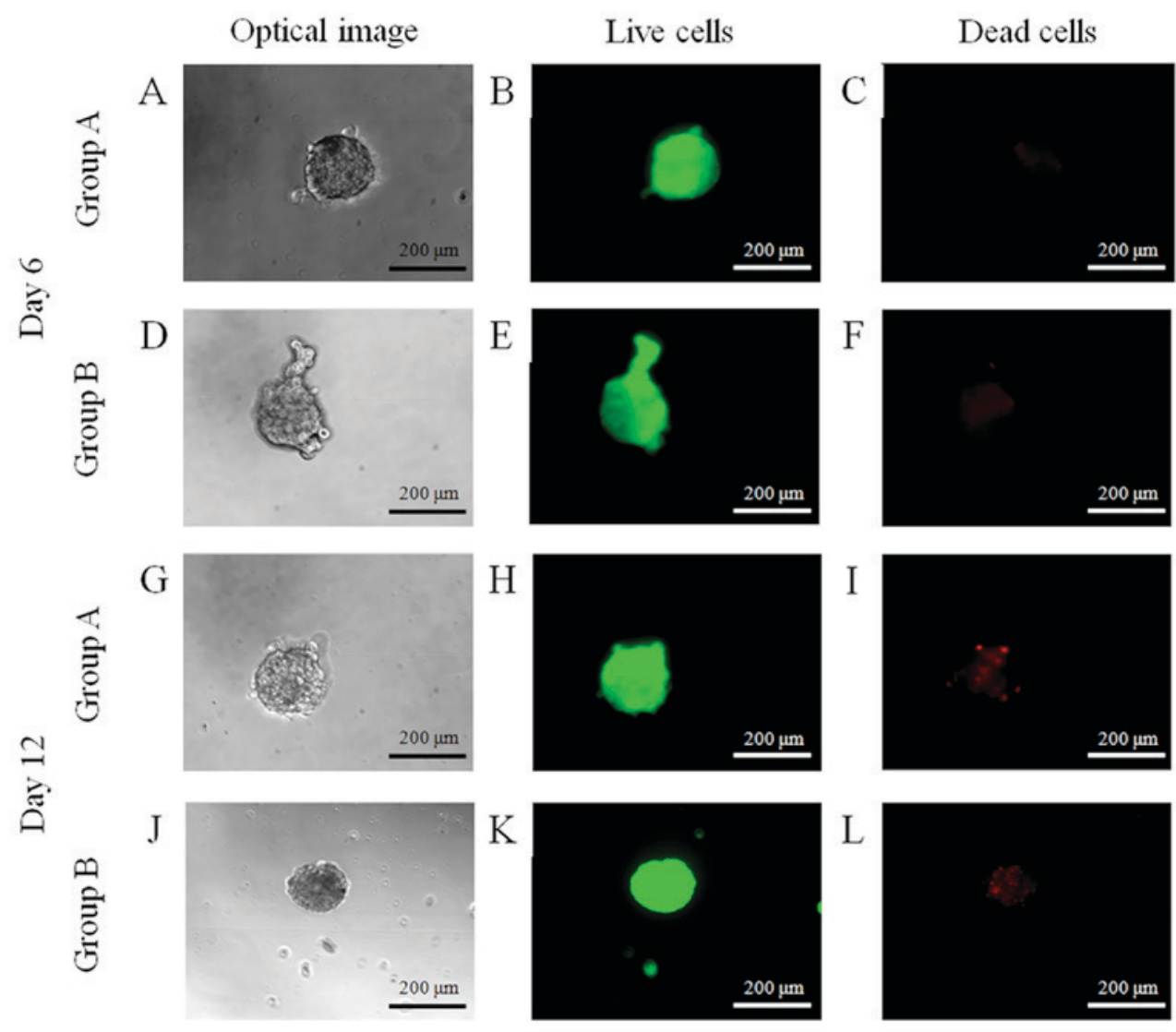

Figure 4. Live/dead cell imaging of spheroids at days 6 and 12. The scale bar indicates $200 \mu \mathrm{m}$ (original magnification, x200). (A-C) Group A at day 6; (D-F) group B at day 6; (G-I) group A at day 12; (J-L) group B at day 12.

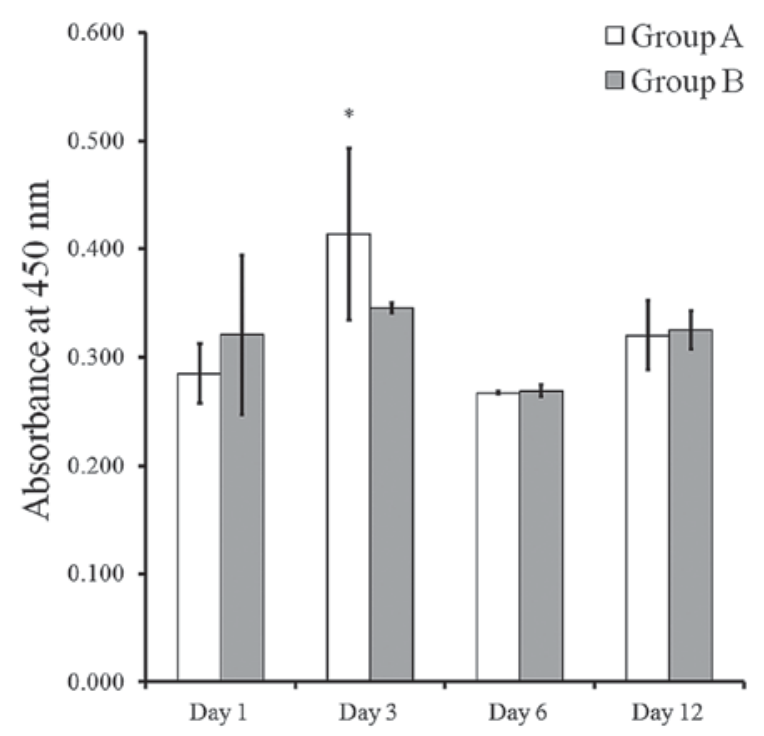

Figure 5. Mean absorbance $( \pm$ standard deviation), indicative of cellular viability on days $1,3,6$, and $12 .{ }^{*} \mathrm{P}<0.05$ vs. group A at day 1.

diameters in group B were $212.0 \pm 19.3$ and $157.1 \pm 27.2 \mu \mathrm{m}$ at days 1 and 3 , respectively.

Determination of cell viability. The majority of cells in the spheroids emitted green fluorescence, and the morphology was round without marked changes at day 6 (Fig. 4). At day 12, the majority of cells in the spheroids emitted green fluorescence; however a small portion of red fluorescence was noted.

The results of cell viability using Cell Counting Kit- 8 following culturing at days $1,3,6$, and 12 are presented in Fig. 5. The cell viability in group B was typically higher than that in group A at each time point, although this did not reach statistical significance $(\mathrm{P}>0.05)$. The Cell Counting Kit-8 assay values of groups A and B at day 1 were $0.285 \pm 0.027$ and $0.320 \pm 0.074$, respectively. The viability values of groups A and $B$ at day 3 were $0.413 \pm 0.080$ and $0.345 \pm 0.005$, respectively. The Cell Counting Kit-8 assay values of groups A and B at day 6 were $0.267 \pm 0.001$ and $0.269 \pm 0.006$, respectively. The Cell Counting Kit- 8 assay value of groups A and B at day 12 were $0.320 \pm 0.032$ and $0.325 \pm 0.018$, respectively.

Maintenance of stemness. Spheroids were stained with NL493-conjugated SSEA-4 (green) and NL557-conjugated TRA-1-60(R) (red) antibodies or with NL493-conjugated SSEA-4 (green) and NL557-conjugated SSEA-1 (red) antibodies (Fig. 6). The spheroids were positive for the stem-cell markers SSEA-4 and TRA-1-60(R), and were negative for SSEA-1, which suggests that these spheroids primarily contained undifferentiated human stem cells.

Osteogenic differentiation. Mineralized extracellular deposits were observed following Alizarin Red S staining at days 14 and 21 (Fig. 7). A marked increase in mineralized deposits was observed in group B compared with group A. The quantitative 


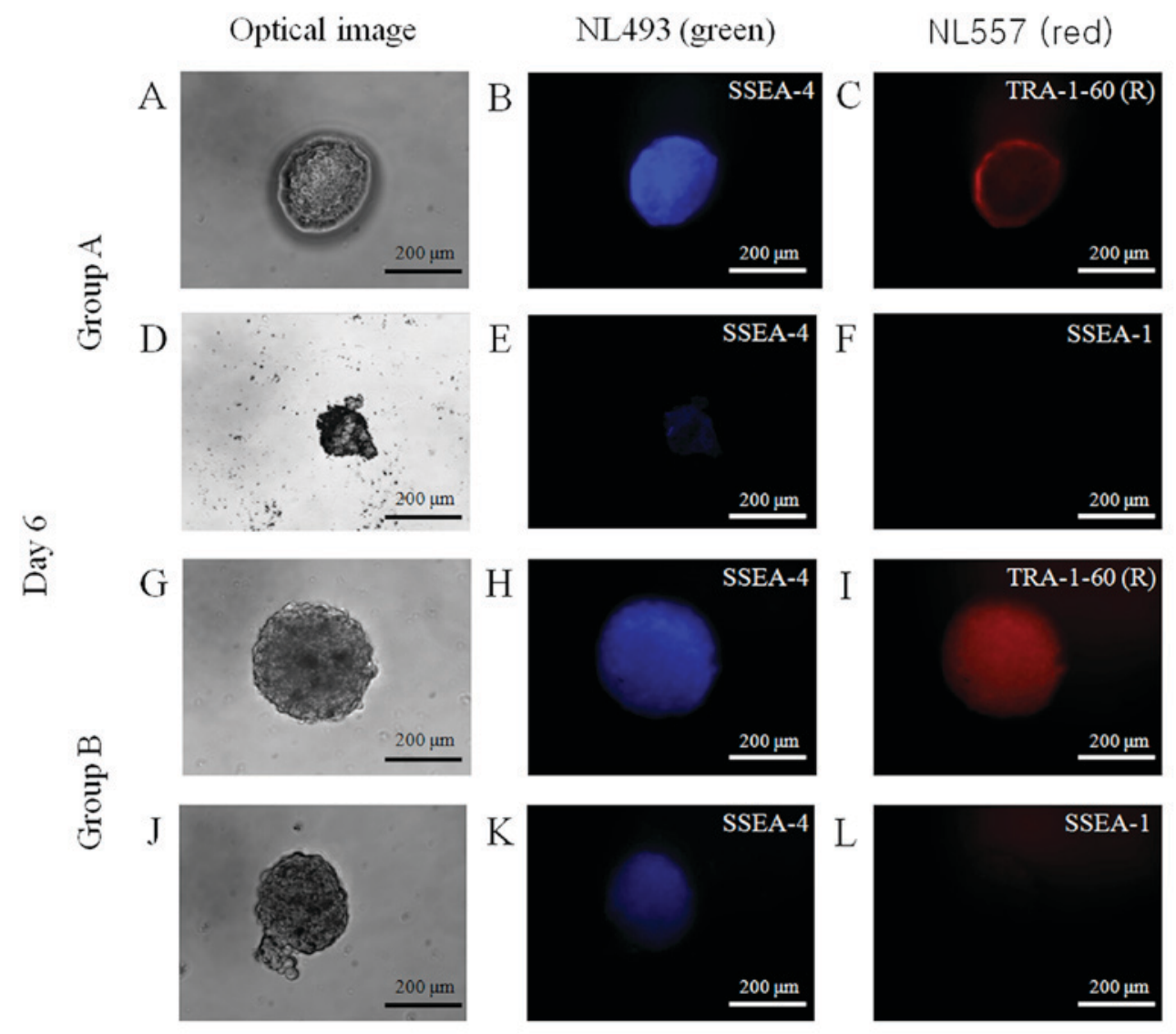

Figure 6. Evaluation of the maintenance of stemness with immunocytochemical staining at day 6. Spheroids were stained with NL493-conjugated SSEA-4 (green), NL557-conjugated TRA-1-60(R) (red) and NL557-conjugated SSEA-1 (red) antibodies. SSEA-4 and TRA-1-60(R) served as positive markers for stem cells, and SSEA-1 was used as a negative marker. The scale bar indicates $200 \mu \mathrm{m}$ (original magnification, x200). (A-F) Group A at day 6; (G-L) group B at day 6.
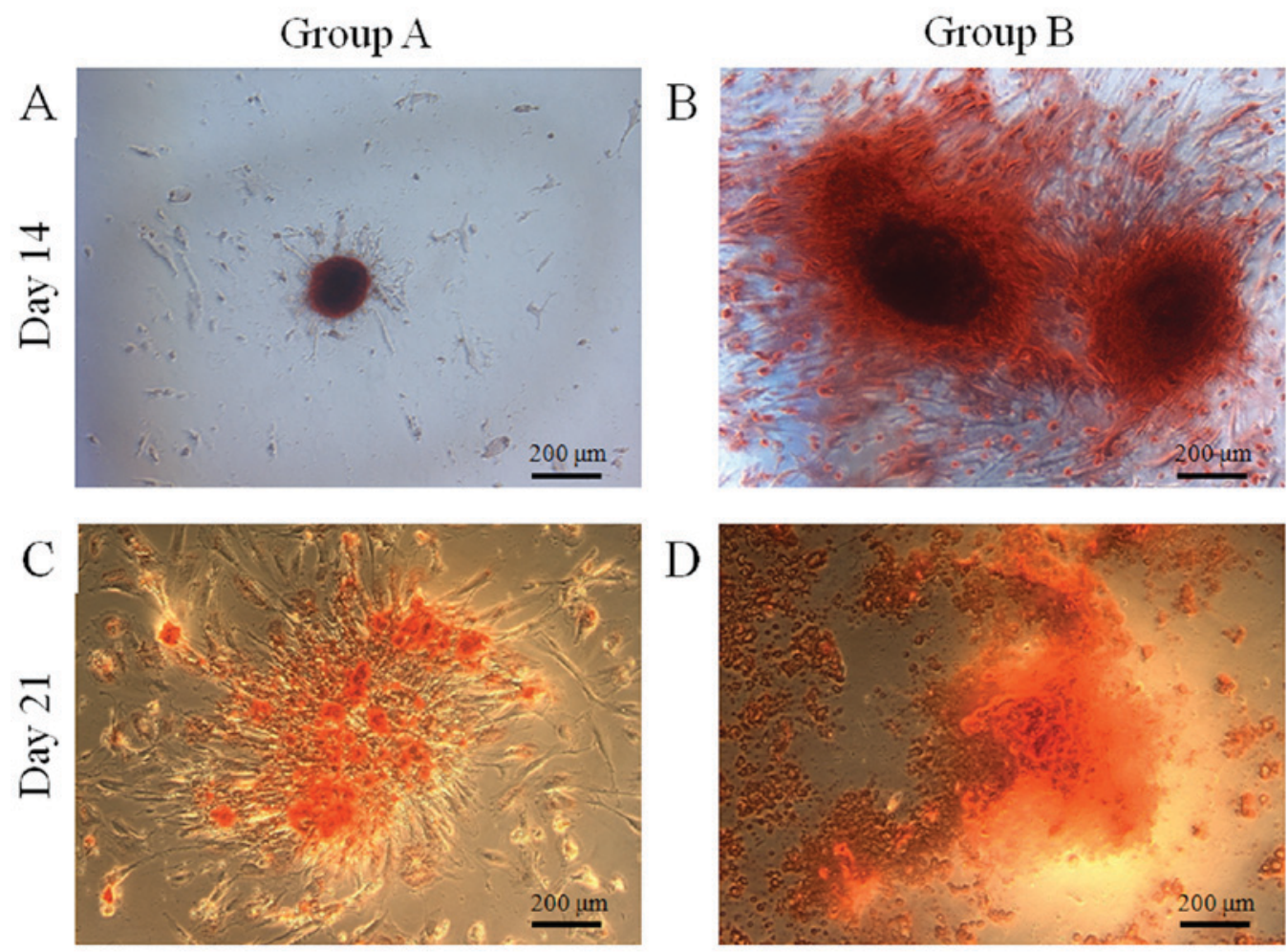

Figure 7. Osteogenic differentiation at days 14 and 21. The scale bar indicates $200 \mu \mathrm{m}$ (original magnification, x100). (A) Group A at day 14; (B) group B at day 14; (C) group A at day 21; (D) group B at day 21. 


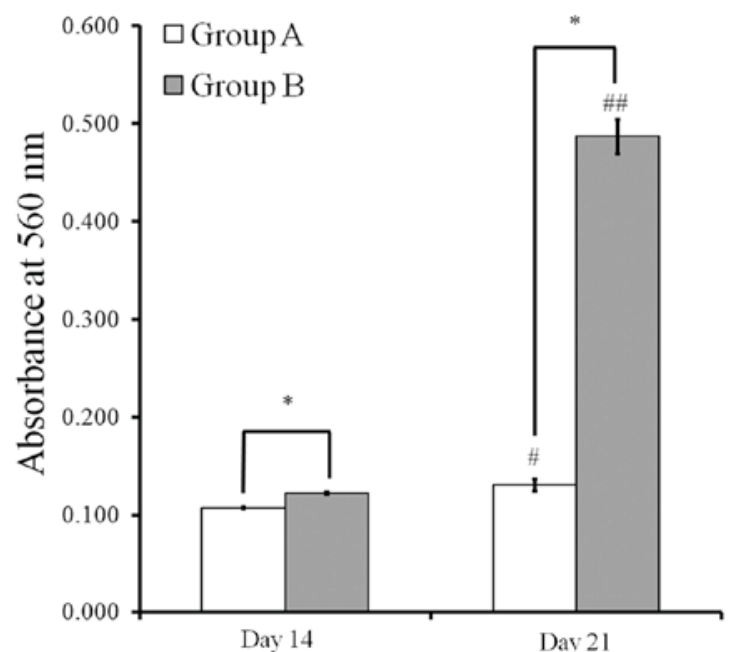

Figure 8. Quantitative results of osteogenic differentiation at days 14 and 21 (mean absorbance \pm standard deviation). ${ }^{*} \mathrm{P}<0.05$; ${ }^{\#} \mathrm{P}<0.05$ vs. group A at day 14 ; ${ }^{\# \#} \mathrm{P}<0.05$ vs. group $\mathrm{B}$ at day 14 .

Group A

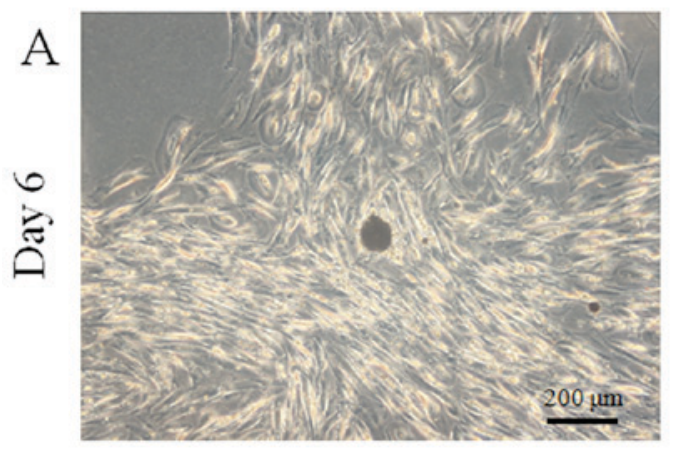

Group B

B

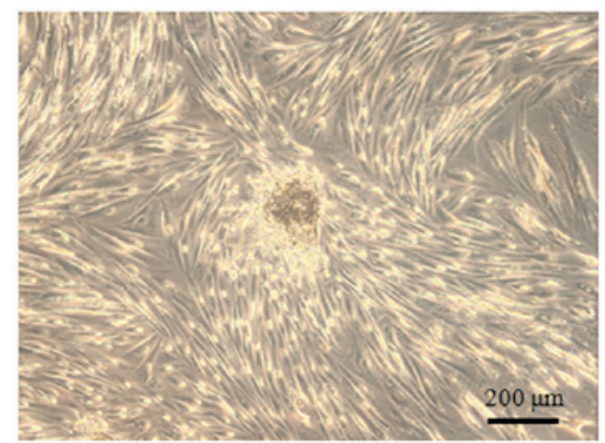

Group B

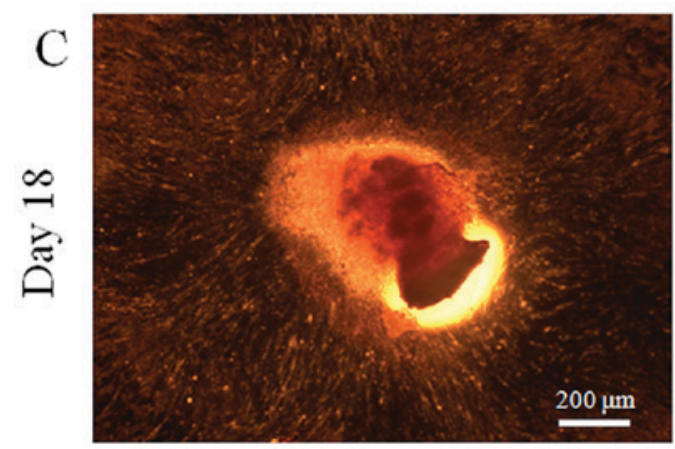

D

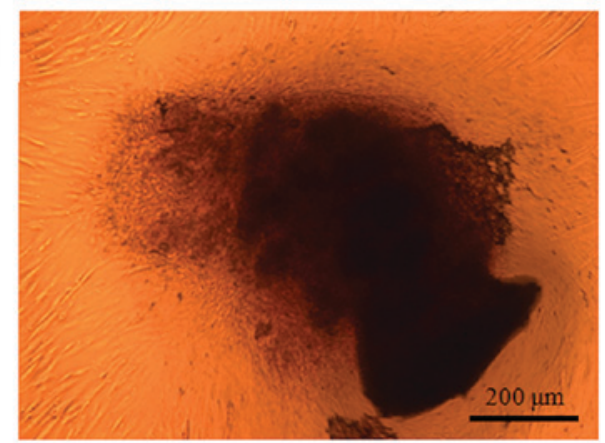

Figure 9. Adipogenic differentiation at days 6 and 18. The scale bar indicates $200 \mu \mathrm{m}$. (A) Group A at day 6 (original magnification, x100); (B) group B at day 6 (original magnification, x100); (C) group B at day 18 (original magnification, x50); (D) group B at day 18 (original magnification, x100).

results regarding bound dye were presented in Fig. 8. Quantitative values at day 14 were $0.107 \pm 0.001$ and $0.122 \pm 0.001$ for groups A and B, respectively. The values at day 21 were $0.130 \pm 0.006$ and $0.487 \pm 0.018$ for groups $A$ and B, respectively. Values for group B were significantly higher than in group A at each point $(\mathrm{P}<0.05)$.

Adipogenic differentiation. The results of adipogenic differentiation are shown in Fig. 9. Oil globules were increased in both groups at day 18 compared with day 6 .
Chondrogenic differentiation. The results of chondrogenic differentiation are shown in Fig. 10. The spheroids were stained with Alcian blue, and staining was more evident at day 18 when compared with day 6 in both groups.

\section{Discussion}

In the present study, the stemness, viability, and differentiation potential of gingiva-derived stem-cell spheroids were maintained during the experimental periods. 
Group A

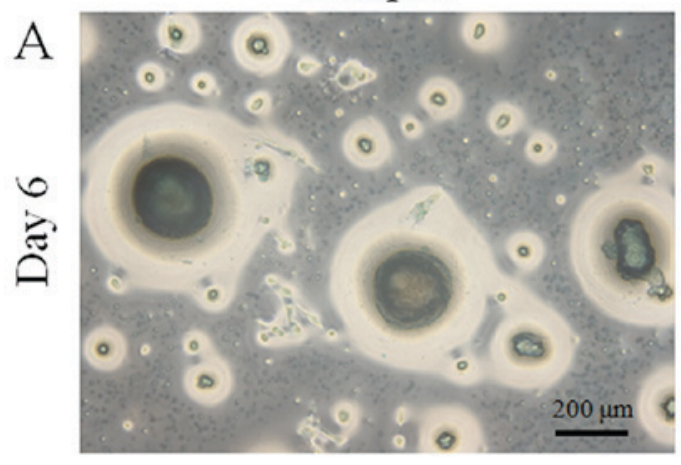

Group B

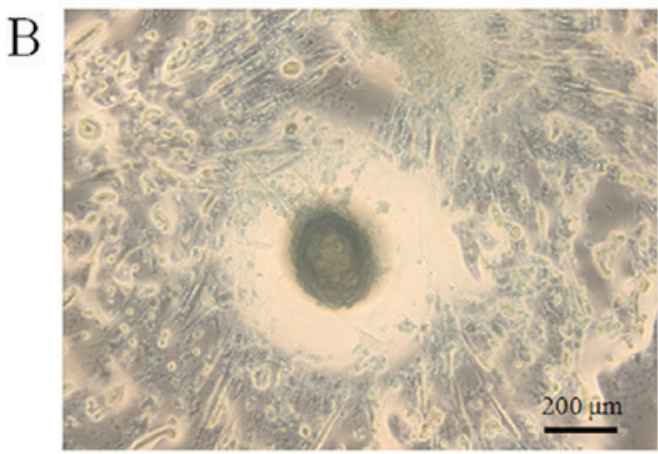

Group A
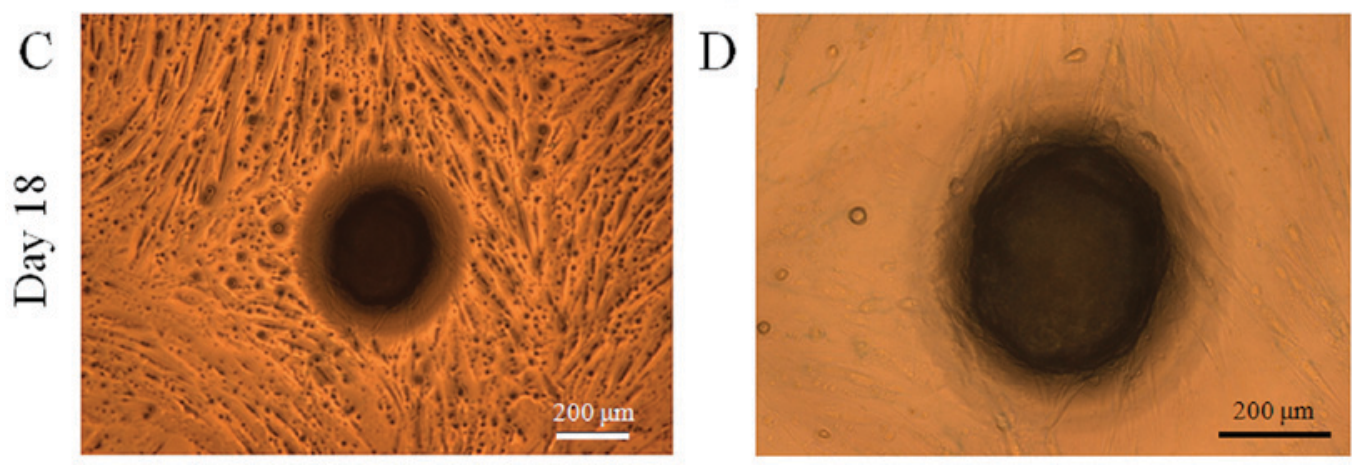

Figure 10. Chondrogenic differentiation at days 6 and 18. The scale bar indicates $200 \mu \mathrm{m}$. (A) Group A at day 6 (original magnification, x100); (B) group B at day 6 (original magnification, x100); (C) group A at day 18 (original magnification, x100); (D) group A at day 18 (original magnification, x200).

Spontaneous cell aggregation only occurs in a fairly limited number of cell lines, and stem cells with self-renewing capacity typically possess spheroid-forming capacity (7). It is possible to generate spheroids in several ways, such as hanging-drop culture, pellet culture, dynamic culture including spinner flask, or rotary cell-culture systems $(4,11,12)$. Microwell culture systems have been developed to generate homogenous stem-cell colonies of defined sizes and shapes, and to study how colony morphology may affect cell fate (13). The authors of the present study have recently demonstrated that stem-cell spheroids may be produced with gingiva-derived stem cells using microwells (14). This method seems convenient for generating cell aggregates without causing shear stress damage (4). The decrease of diameter in spheroids may be explained by the increased cell-cell contact from neighboring cells in three-dimensional culture $(5,15)$.

Accumulating evidence has suggested that the cellular microenvironment has an important role in determining stemness properties $(4,16,17)$. The present study demonstrated that the spheroids maintained viability and stemness during the experimental period using polydimethylsiloxane-based concave micromolds. It has also been demonstrated in previous that the aggregation of adult human mesenchymal stem cells to produce three-dimensional cellular spheroids helped to maintain the expression of stemness marker genes in cells, which was reiterated by the present findings $(4,6)$. A recent study has also indicated that rabbit corneal stromal-cell-derived spheroids positively expressed mesenchymal and stem-cell phenotypes, which were immunopositive for vimentin and cluster of differentiation 34 (a mesenchymal cell marker and a stem cell marker, respectively), as well as the mRNA expression of nestin and Nanog (a neural stem cell marker and a stem cell marker, respectively) (7).

The present study study also demonstrated that viability was maintained during the experimental period. It was recently suggested that a spheroid culture of mesenchymal stem cells may meet the requirement of adherent growth and improve culture efficiency (3). A recent report showed that compact cellular spheroids were formed with adipose-derived stem cells, and spheroids remained viable when cultured in a microgravity bioreactor (4).

The present study clearly showed that osteogenic, adipogenic, and chondrogenic differentiation were present within the spheroids. The increase of differentiation was noted with an increase in the number of cells. Enhanced chondrogenic differentiation potential of human gingival fibroblasts was previously evaluated via spheroid formation on chitosan membranes (18).

Conclusively, stem-cell spheroids were demonstrated to form gingival cells which maintained stemness, viability, and differentiation potential during the experimental period. These findings suggest that this convenient method may be applied as a promising strategy for stem-cell therapy.

\section{Acknowledgements}

The present study was supported by Basic Science Research Program through the National Research Foundation of Korea, funded by the Ministry of Science, Information and Communication Technology and Future Planning (grant no. NRF-2014R1A1A1003106). 


\section{References}

1. Park JB, Bae SS, Lee PW, Lee W, Park YH, Kim H, Lee K and Kim I: Comparison of stem cells derived from periosteum and bone marrow of jaw bone and long bone in rabbit models. Tissue Eng Regen Med 9: 224-230, 2012.

2. Park JB, Lee KS, Lee W, Kim HS, Lee KH and Kim IS: Establishment of the chronic bone defect model in experimental model mandible and evaluation of the efficacy of the mesenchymal stem cells in enhancing bone regeneration. Tissue Eng Regen Med 10: 18-24, 2013.

3. Li Y, Guo G, Li L, Chen F, Bao J, Shi YJ and Bu H: Three-dimensional spheroid culture of human umbilical cord mesenchymal stem cells promotes cell yield and stemness maintenance. Cell Tissue Res 360: 297-307, 2015.

4. Zhang S, Liu P, Chen L, Wang Y, Wang Z and Zhang B: The effects of spheroid formation of adipose-derived stem cells in a microgravity bioreactor on stemness properties and therapeutic potential. Biomaterials 41: 15-25, 2015.

5. Hsiao C, Tomai M, Glynn J and Palecek SP: Effects of 3D microwell culture on initial fate specification in human embryonic stem cells. AIChE J 60: 1225-1235, 2014.

6. Jiang CF, Hsu SH, Tsai KP and Tsai MH: Segmentation and tracking of stem cells in time lapse microscopy to quantify dynamic behavioral changes during spheroid formation. Cytometry A 87: 491-502, 2015.

7. Li H, Dai Y, Shu J, Yu R, Guo Y and Chen J: Spheroid cultures promote the stemness of corneal stromal cells. Tissue Cell 47: $39-48,2015$.

8. Jin SH, Lee JE, Yun JH, Kim I, Ko Y and Park JB: Isolation and characterization of human mesenchymal stem cells from gingival connective tissue. J Periodontal Res 50: 461-467, 2015.

9. Jin SH, Kweon H, Park JB and Kim CH: The effects of tetracycline-loaded silk fibroin membrane on proliferation and osteogenic potential of mesenchymal stem cells. J Surg Res 192: e1-e9, 2014.
10. Park JB, Kim YS, Lee G, Yun BG and Kim CH: The effect of surface treatment of titanium with sand-blasting/acid-etching or hydroxyapatite-coating and application of bone morphogenetic protein-2 on attachment, proliferation, and differentiation of stem cells derived from buccal fat pad. Tissue Eng Regen Med 10: 115-121, 2013.

11. Mehta G, Hsiao AY, Ingram M, Luker GD and Takayama S: Opportunities and challenges for use of tumor spheroids as models to test drug delivery and efficacy. J Control Release 164: 192-204, 2012

12. Page H, Flood P and Reynaud EG: Three-dimensional tissue cultures: Current trends and beyond. Cell Tissue Res 352: 123-131, 2013.

13. Hsiao C and Palecek SP: Microwell regulation of pluripotent stem cell self-renewal and differentiation. Bionanoscience 2: 266-276, 2012.

14. Lee SI, Yeo SI, Kim BB, Ko Y and Park JB: Formation of size-controllable spheroids using gingiva-derived stem cells and concave microwells: Morphology and viability tests. Biomed Rep 4: 97-101, 2016.

15. Azarin SM, Larson EA, Almodovar-Cruz JM, de Pablo JJ and Palecek SP: Effects of 3D microwell culture on growth kinetics and metabolism of human embryonic stem cells. Biotechnol Appl Biochem 59: 88-96, 2012.

16. Gattazzo F, Urciuolo A and Bonaldo P: Extracellular matrix: A dynamic microenvironment for stem cell niche. Biochim Biophys Acta 1840: 2506-2519, 2014.

17. Wan PX, Wang BW and Wang ZC: Importance of the stem cell microenvironment for ophthalmological cell-based therapy. World J Stem Cells 7: 448-460, 2015.

18. Hsu SH, Huang GS, Lin SY, Feng F, Ho TT and Liao YC: Enhanced chondrogenic differentiation potential of human gingival fibroblasts by spheroid formation on chitosan membranes. Tissue Eng Part A 18: 67-79, 2012. 